\title{
Pen Dosing Unit
}

National Cancer Institute

\section{Source}

National Cancer Institute. Pen Dosing Unit. NCI Thesaurus. Code C149748.

A unit of presentation used to represent the quantity of product that is found in a single pen container. 